\title{
Title: Survey of peridomestic mammal susceptibility to SARS-CoV-2 infection
}

2

Authors: Angela M. Bosco-Lauth, J. Jeffrey Root, Stephanie M. Porter, Audrey E. Walker, Lauren Guilbert, Daphne Hawvermale, Aimee Pepper, Rachel M. Maison, Airn E. Hartwig, Paul Gordy, Helle Bielefeldt-Ohmann, Richard A. Bowen.

\section{Affiliations:}

Colorado State University, Fort Collins, Colorado, USA (A. Bosco-Lauth, S. Porter, A. Walker, L Guilbert, D. Hawvermale, A. Pepper, R. Maison, A. Hartwig, P. Gordy, R. Bowen.)

U.S. Department of Agriculture, Animal and Plant Health Inspection Service, Wildlife Services, National Wildlife Research Center, Fort Collins, Colorado, USA. (J. Root)

School of Chemistry \& Molecular Biosciences and Australian Infectious Diseases Research Centre, The University of Queensland. St Lucia, Queensland, Australia (H. Bielefeldt-Ohmann)

Keywords: Coronavirus, cottontail rabbit, deer mouse, experimental infection, fox squirrel, ground squirrel, house mouse, peridomestic, mesocarnivore, raccoon, rodent, SARS-CoV-2, striped skunk, wildlife 


\section{Abstract}

23 Wild animals have been implicated as the origin of SARS-CoV-2, but it is largely unknown how

24 the virus affects most wildlife species and if wildlife could ultimately serve as a reservoir for

25 maintaining the virus outside the human population. Here we show that several common

26 peridomestic species, including deer mice, bushy-tailed woodrats, and striped skunks, are

27 susceptible to infection and can shed the virus in respiratory secretions. In contrast, we

28 demonstrate that cottontail rabbits, fox squirrels, Wyoming ground squirrels, black-tailed prairie

29 dogs, house mice, and racoons are not susceptible to SARS-CoV-2 infection. Our work expands

30 upon the existing knowledge base of susceptible species and provides evidence that human-

31 wildlife interactions could result in continued transmission of SARS-CoV-2.

\section{Introduction}

The rapid global expansion of severe acute respiratory syndrome (SARS) coronavirus 2 infection(s) were potentially linked to wild animals in a wet market (1), human-to-human transmission is currently the dominant mechanism of viral spread. Peridomestic animals, which are represented by wild and feral animals living within close proximity to humans, represent key species to evaluate for SARS-CoV-2 epidemiology for multiple reasons. First, given their common associations with humans and anthropogenically modified habitats, they represent the

42 wildlife species with the greatest chance of exposure to the virus from humans (i.e., reverse

43 zoonosis) or pets such as cats. Second, should select peridomestic wildlife prove to be

44 susceptible to the virus and have the capacity to replicate it to high viral titers, these species 
45 would have the potential to maintain the virus among conspecifics. Third, should some species

46 possess the maintenance host criteria mentioned above, they would represent wildlife species

47 that would have the greatest chance (e.g., shedding ability and proximity to humans) to spread

48 the virus back to humans. Wild rodents, cottontail rabbits (Sylvilagus sp.), raccoons (Procyon

49 lotor) and striped skunks (Mephitis mephitis) can exhibit peridomestic tendencies in urban and

50 suburban environments. Members of all these species/taxonomic groups have been shown to

51 shed influenza A viruses following experimental inoculations $(2,3,4)$, suggesting they might

52 harbor productive infections when exposed to other human-pathogenic respiratory viruses.

Based upon protein analyses of amino acid residues of ACE2, TMPRSS2 and S protein,

55 species susceptibility analyses suggested that, among other taxonomic groups, both carnivores

56 and wild rodents are potentially high-risk groups $(5,6,7)$. Predicting specific species'

57 susceptibility, however, is more challenging. Looking at protein sequence analysis of ACE2

58 binding with the S protein of SARS-CoV-2, one study indicated that raccoons could be ruled out

59 as potential hosts for SARS-CoV-2 (6) and a different study based upon sequence analysis

60 suggested that the western spotted skunks (Spilogale gracilis) had a very low prediction of

61 SARS-CoV-2 S-binding propensity (7). Similarly, the same study also suggested that American

62 mink (Neovison vison) have a similar prediction as western spotted skunks (7). However, over

63 the last several months, outbreaks of SAR-CoV-2 in commercial mink farms have been noted in

64 Europe and more recently in the U.S. $(8,9)$. Respiratory problems, rapid transmission, and/or

65 unusually high mortality have been noted in this species in various regions $(9,10)$, which

66 suggests that the aforementioned analyses have limitations. 
Rodents are the largest and most diverse order of mammals, so it is unsurprising that the susceptibility of rodents to SARS-CoV-2 varies by species. To date, only a handful of rodent species have been evaluated as potential reservoir hosts or animal models for SARS-CoV-2, and

71 the results largely indicate that outbred species, including lab animals, are at most only

72 moderately affected. Most non-transgenic laboratory mice (Mus musculus) are resistant to

73 infection, while transgenic humanized mice and hamsters, including Syrian hamsters

74 (Mesocricetus auratus) and dwarf hamsters (Phodopus sp.), are highly susceptible, with at least

75 one report of Roborovky’s dwarf hamsters becoming fatally diseased within three days of

76 exposure $(11,12,13)$. Other species, including deer mice (Peromyscus maniculatus), become

77 infected and shed low titers of virus, but the infection is subclinical $(14,15)$. Considering that

78 there are more than 1700 species of rodents world-wide, many of which exist closely at the

79 human-wildlife interface, there remain many unanswered questions about SARS-CoV-2 and wild 80 rodents. prime position to come into contact with SARS-CoV-2 infected humans. In one study, New

84 Zealand white rabbits were experimentally infected and shed infectious virus for up to seven 85 days without signs of clinical disease (16) Wild rabbits, particularly cottontails in the U.S., are 86 prolific and commonly found around human dwellings, farms, and commercial buildings.

87 Further, as with rodents, wild rabbits are highly likely to be predated upon by domestic cats.

88 Thus, determining the susceptibility of these animals is critically important to interpreting the risk posed to them and by them from infection with SARS-CoV-2. 
Among carnivores, felids and mustelids have been frequently linked to SARS-CoV-2

91 infections since the early stages of the pandemic. Domestic cats are highly susceptible to SARS-

92 CoV-2 and are capable of transmitting the virus to other cats, suggesting that they could

93 potentially transmit to other animals as well $(17,18)$. While striped skunks are currently

94 considered to be mephitids, they are highly related to mammals within the family mustelidae and

95 were formerly classified as mustelids. Thus, based on the findings of SARS-CoV-2

96 susceptibility in various mustelids, the closely related mephitids are a logical candidate to

97 evaluate for the replication of this virus. Raccoons are notoriously associated with human

98 environments and frequently interact with human trash and sewage, which has been proposed as

99 a potential indirect means for infected humans to transmit SARS-CoV-2 to mammalian wildlife

100 (e.g., raccoons and select mustelids) $(19,20,21)$. Thus, it is important to determine the relative

101 susceptibility of these common peridomestic carnivores and assess the likelihood that they could

102 propagate infection.

103

In this study, we assessed six common peridomestic rodent species for susceptibility to

105 SARS-CoV-2: deer mice, wild-caught house mice (Mus musculus), bushy-tailed woodrats (aka

106 "pack rats"; Neotoma cinerea), fox squirrels (Sciurus niger), Wyoming ground squirrels

107 (Urocitellus elegans), and black-tailed prairie dogs (Cynomys ludovicianus). These rodents are

108 common in many parts of the United States, several of them frequently come into close contact

109 with humans and human dwellings, and some are highly social animals, thus increasing the

110 likelihood of pathogen transmission among conspecifics. In addition, we evaluated three other

111 common peridomestic mammals: cottontail rabbits, raccoons, and striped skunks. Our results

112 indicate that $33 \%(3 / 9)$ of the species evaluated are susceptible to SARS-CoV-2 infection, 
113 suggesting that wildlife may become critically implicated in the continued persistence of the

114 virus.

116 Materials and Methods

118 Animals

119 The following mixed-sex animals were evaluated for susceptibility to SARS-CoV-2: Deer mice,

120 house mice, bushy-tailed woodrats, Wyoming ground squirrels, black-tailed prairie dogs, fox

121 squirrels, cottontail rabbits, striped skunks, and raccoons. Deer mice, house mice and bushy-

122 tailed woodrats were trapped using Sherman traps baited with grain. Wyoming ground squirrels,

123 fox squirrels, black-tailed prairie dogs, and cottontails were trapped using Tomahawk live traps

124 (e.g., 7 × 7 × 20 or 7 × 7 × 24). All trapping was done in Northern Colorado (Larimer, Jackson

125 and Weld counties) in accordance with Colorado wildlife regulations and with appropriate

126 permits in place. Skunks and raccoons were purchased from a private vendor. Animals were

127 housed in an Animal Biosafety Level-3 (ABSL3) facility at Colorado State University, in

128 12'x18' rooms with natural lighting and controlled climate. Mice, black-tailed prairie dogs, and

129 Wyoming ground squirrels were group housed by species with ad libitum access to water and

130 food. All other animals were housed individually with access to food and water ad libitum.

131 Rodents were maintained on Teklad ${ }^{\circledR}$ Rodent Diet (Enviro, Madison, WI) supplemented with

132 fresh fruit and occasional nuts. Rabbits were fed alfalfa pellets (Manna Pro ${ }^{\circledR}$ Corp, Denver,

133 Colorado) supplemented with grass hay and apples. Skunks and raccoons were maintained on

134 Mazuri ${ }^{\circledR}$ Omnivore Diet (Mazuri Exotic Animal Nutrition, St. Louis, MO) supplemented with

135 fresh fruit and occasional eggs. Raccoons, striped skunks and black-tailed prairie dogs were 
136 implanted with thermally-sensitive microchips (Bio-Thermo Lifechips, Destron-Fearing) for

137 identification and temperature measurement, deer mice were ear notched; all other animals were

138 identified by cage number or distinct markings.

139 Virus

140 SARS-CoV-2 virus strain WA1/2020WY96 was obtained from BEI Resources (Manassas, VA,

141 USA), passaged twice in Vero E6 cells and stocks frozen at $-80^{\circ} \mathrm{C}$ in Dulbecco's Modified Eagle

142 Medium (DMEM) with 5\% fetal bovine serum and antibiotics. Virus stock was titrated on Vero

143 cells using standard double overlay plaque assay (17) and plaques were counted 72 hours later to

144 determine plaque-forming units (pfu) per ml.

146 Virus challenge

147 Prior to challenge with SARS-CoV-2, most animals were lightly anesthetized as needed with 1-3

$148 \mathrm{mg} / \mathrm{kg}$ xylazine and 10-30 mg/kg ketamine hydrochloride (Zetamine ${ }^{\mathrm{TM}}$ ) and a blood sample

149 collected just before inoculation (Day 0). Virus diluted in phosphate buffered saline (PBS) was

150 administered to all species via pipette into the nares (50ul for deer and house mice, $100 \mathrm{ul}$ for

151 bushy-tailed woodrats, and 200ul for all other species) and animals were observed until fully

152 recovered from anesthesia. Virus back-titration was performed on Vero cells immediately

153 following inoculation, confirming that animals received between 4.5 and $4.9 \log _{10}$ pfu of SARS-

154 CoV-2.

156 Sampling

157 Groups of three animals from each species (two for ground squirrels) were used for preliminary

158 studies to evaluate viral shedding and acute pathological changes. For these animals, oral swabs 
159 were obtained pre-challenge and on days 1-3 post-challenge, at which time animals were

160 euthanized and the following tissues harvested for virus isolation and formalin fixation: trachea,

161 nasal turbinates, lung, heart, liver, spleen, kidney, small intestine, and olfactory bulb. The

162 exception to this was raccoons, for which only one animal was euthanized at day 3; the

163 remaining two were kept through day 28 to evaluate serological response. The remaining 3-6

164 animals per select species were swabbed daily from days 0-5 and 7 to further evaluate duration

165 of viral shedding (if any). Striped skunks and raccoons were sedated for all sampling and a nasal

166 swab was collected in addition to the oral swab. Tissues harvested from animals euthanized on

167 day 7 were evaluated as for the day 3 animals. The remaining animals were euthanized at 28

168 days post-infection (DPI) and tissues were harvested for histopathology and serum was collected

169 for serology. Table 1 illustrates the necropsy scheme for each species.

171 Table 1. Wildlife species evaluated for experimental infections with SARS-CoV-2 and day post

172 infection the animals were euthanized.

\begin{tabular}{llll}
\hline Animals & \# euthanized at 3 & \# euthanized at 7 DPI & \# euthanized at 28 \\
& DPI* & DPI \\
\hline Deer mice $(\mathrm{n}=9)$ & 3 & 3 & 3 \\
House mice $(\mathrm{n}=6)$ & 3 & 0 & 3 \\
Bushy-tailed & 3 & 0 & 3 \\
woodrats(n=6) & & & 0 \\
Fox squirrels (n=3) & 3 & 0 & 0 \\
Wyoming ground & 2 & 0 & \\
squirrels (n=2) & & & 0 \\
\hline
\end{tabular}




$\begin{array}{llcc}\text { Black-tailed prairie } & 3 & 3 & 3 \\ \operatorname{dogs}(\mathrm{n}=9) & 0 & 0 \\ \text { Cottontails }(\mathrm{n}=3) & 3 & 0 & 2 \\ \text { Raccoons }(\mathrm{n}=3) & 1 & 0 & 3 \\ \text { Striped skunks }(\mathrm{n}=6) & 3 & \end{array}$

\footnotetext{
*Table footnotes: ${ }^{*}$ DPI = Days post-infection
}

173

174

175

176 Clinical evaluations were performed for all animals daily and included assessment for

177 temperament and presence of any clinical signs of disease, such as ocular discharge, nasal

178 discharge, ptyalism, coughing/sneezing, dyspnea, diarrhea, lethargy, anorexia, and if moribund.

179 The stress of handling wild animals for sampling precluded the ability to obtain accurate body

180 temperature measurements; as such, temperature was excluded in these preliminary studies for

181 all species except skunks and raccoons, which were implanted with thermal microchips and

182 could be measured under sedation during sampling.

184 Viral assays

185 Virus isolation was performed on all oral swab, nasal swab and 3 DPI tissue samples by double

186 overlay plaque assay on Vero cells as previously described (17). Briefly, 6-well plates with

187 confluent monolayers of cells were washed once with PBS and inoculated with $100 \mu$ of serial

188 10-fold dilutions of samples, incubated for 1 hour at $37^{\circ} \mathrm{C}$, and overlaid with a $0.5 \%$ agarose in

189 MEM containing 2\% fetal bovine serum and antibiotics/antifungal agents. A second overlay with 
neutral red dye was added at 48 hours and plaques were counted at 72 hours. Viral titers were

191 reported as the $\log _{10}$ pfu per swab (oropharyngeal/nasal) or per gram (tissue).

193 Serology

194 Plaque reduction neutralization assays (PRNT) were performed as previously described (17).

195 Serum was heat-inactivated for $30 \mathrm{mins}$ at $56^{\circ} \mathrm{C}$, and two-fold dilutions prepared in BA-1 (Tris-

196 buffered MEM containing 1\% bovine serum albumin) starting at a 1:5 dilution were aliquoted

197 onto 96-well plates. An equal volume of virus was added to the serum dilutions and incubated

198 for 1 hour at $37^{\circ} \mathrm{C}$. Following incubation, serum-virus mixtures were plated onto Vero

199 monolayers as described for virus isolation assays. Antibody titers were recorded as the

200 reciprocal of the highest dilution in which $>90 \%$ of virus was neutralized.

201

$202 q R T-P C R$

203 Plaques were picked from culture plates from each positive animal to confirm SARS-CoV-2

204 viral shedding. RNA extractions were performed per the manufacturer's instructions using

205 Qiagen QiaAmp Viral RNA mini kits. RT-PCR was performed as recommended using the

206 E_Sarbeco primer probe sequence as described by Corman and colleagues (22) and the

207 Superscript III Platinum One-Step qRT-PCR system (Invitrogen), with the following

208 modification: the initial reverse transcription was at $50^{\circ} \mathrm{C}$. RNA standards for PCR were obtained

209 from BEI Resources (Manassas, VA, USA).

210

211 Histopathology 
212 Animal tissues were fixed in 10\% neutral-buffered formalin for 12 days and transferred to $70 \%$

213 ethanol prior to processing for paraffin-embedding, sectioning for H\&E staining. Slides were

214 read by a veterinary pathologist blinded to the treatments.

\section{Results}

\section{Viral shedding}

219 Of the nine species evaluated, three (deer mice, bushy-tailed woodrats, and striped skunks) shed infectious virus following challenge (Figure 1). Deer mice, which have previously been up to four days and virus was isolated from lungs $(n=3 / 3)$ and trachea $(n=2 / 3)$ from animals

224 following infection, with peak titers of $3.1 \log _{10} \mathrm{pfu} / \mathrm{swab}$. Bushy-tailed woodrats shed virus orally for up to five days post inoculation $(n=6 / 6)$ and virus was isolated from turbinates $(n=2 / 3)$, trachea $(n=1 / 3)$ and lung $(n=1 / 3)$ from animals necropsied on 3 DPI. Peak titers from bushy-

227 tailed woodrats reached $3.0 \log _{10} \mathrm{pfu} / \mathrm{swab}$ by 3 DPI. Interestingly, the single bushy-tailed

231 time three of the six infected animals shed orally, nasally, or both, with one animal shedding up to 7 DPI. Of the three skunks necropsied on 3 DPI, two had infectious virus in the turbinates, but not in other tissues tested. One of those two animals had $3.2 \log _{10} \mathrm{pfu} / \mathrm{gram}$ in the turbinates but 
235 slightly higher in nasal samples compared to oral, but overall peak titers in skunks were

236 relatively low, with oral titers reaching $2 \log _{10} \mathrm{pfu} / \mathrm{swab}$ and nasal flush titers at $2.3 \log _{10}$

$237 \mathrm{pfu} / \mathrm{swab}$. All animals with plaque-assay positive samples were confirmed for SARS-CoV-2 by

238 RT-PCR. Similarly, all animals that were negative on plaque assay were confirmed negative for

239 viral shedding by RT-PCR.

241 Figure 1: Oropharyngeal shedding of SARS-CoV-2 in deer mice (A), bushy-tailed woodrats (B)

242 and striped skunks (C) and nasal shedding in striped skunks (D). Values expressed as $\log _{10}$

$243 \mathrm{pfu} / \mathrm{swab}$; limit of detection $1 \log _{10} \mathrm{pfu}$.

A Oropharnygeal Shedding in Deer Mice

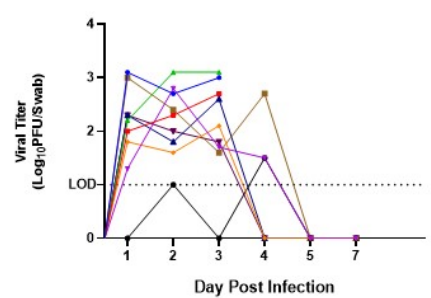

$\mathrm{C}$

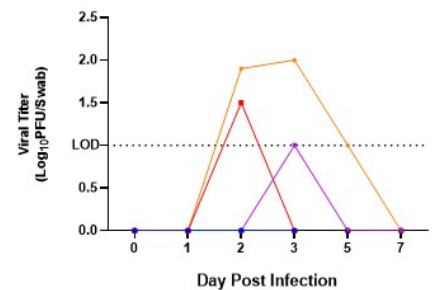

Oropharnygeal Shedding in Woodrats

$\rightarrow$ Mouse 1

$\rightarrow$ Mouse 2

- Mouse 3

$\rightarrow$ Mouse 4

-Mouse 5

- Mouse 6

- Mouse 7

+ Mouse 8

$\rightarrow$ Mouse 9

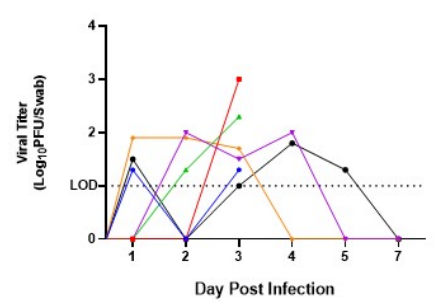

- Rat 1

- Rat 2

- Rat 3

- Rat 4

- Rat 5

$\rightarrow$ Rat 6

$$
\begin{aligned}
& \text { - Skunk } 1 \\
& \text { - Skunk } 2 \\
& \text { - Skunk } 3 \\
& \text { - Skunk } 4 \\
& \text { - Skunk } 5 \\
& \text { - Skunk } 6
\end{aligned}
$$

D Nasal Shedding in Skunks

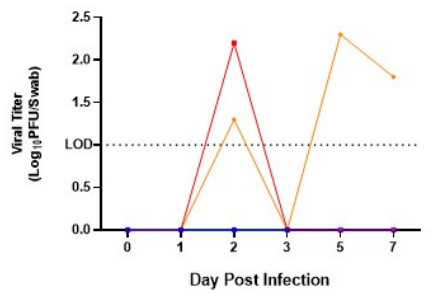

$\rightarrow$ Skunk 1

- Skunk 2

- Skunk 3

- Skunk 4

- Skunk 5

- Skunk 6

\section{Seroconversion}

246 All animals were seronegative against SARS-CoV-2 at the time of inoculation $(<50 \%$ viral

247 neutralization at 1:10 serum dilution). Based on the lack of evidence of infection and the overall 
249 additional time to assess seroconversion. Neutralizing antibody titers were assessed in all

250 animals euthanized at 28 DPI, which included deer mice, house mice, bushy-tailed woodrats,

251 black-tailed prairie dogs, raccoons and striped skunks. All species which had detectable viral

252 infections (deer mice, skunks, and bushy-tailed woodrats) also developed neutralizing antibodies,

253 while the other species (house mice, raccoons, and black-tailed prairie dogs) did not. Deer mice

254 and bushy-tailed woodrats reached or exceeded titers of 1:80, and the two skunks that shed

255 infectious virus reached or exceeded titers of 1:160, while the single skunk that did not shed

256 virus had a titer of 1:10 at 28 DPI. Animals euthanized at 3 DPI were not tested for

257 seroconversion as previous investigations have demonstrated that neutralizing antibodies are

258 typically not detectable during acute infection (23).

\section{Clinical disease}

261 None of the animals exhibited clinical signs of disease (see methods for symptoms) at any time

262 during the study. Skunks and raccoons, which were sedated for procedures which involved

263 sampling, failed to display elevated temperatures at those times. In addition to clinical signs,

264 behavior was monitored by observing animals through double-paned glass and assessing eating

265 and response to provided enrichment (playing with toys, eating treats, using hides, etc.), and

266 none of the animals were observed to behave abnormally following infection when compared to

267 the acclimation period.

\section{Pathology}

270 None of the animals had gross lesions at the time of necropsy. On histopathologic examination,

271 rare, small foci of mild macrophage and neutrophil infiltration were noted in the lungs of two 
272 woodrats and two deer mice with one of the latter also having mild vasculitis. Two skunks

273 presented with well-developed bronchiole associated lymphoid tissue (BALT), but inflammation

274 was not apparent in the lungs or other tissues.

275

276 Conclusions not only susceptible to infection, but can develop fulminating fatal disease $(10,26)$. In contrast, ferrets, which are closely related to mink, shed virus following infection but the infection is

284 subclinical (27). Raccoon dogs, which were heavily implicated in the SARS-CoV-1 outbreak in 2004, are susceptible to SARS-CoV-2 infection, but remain subclinical (28). Experimentally,

286 deer mice can be infected and shed the virus via oral secretions, as demonstrated by this study 287 and others $(14,15)$. However, other mice, including wild house mice and non-transgenic laboratory strains of this species, are not susceptible to infection by SARS-CoV-2 (29). Studies in which bats and select small mammals were experimentally exposed to SARS-CoV-2 show that some species (i.e., fruit bats [Rousettus aegyptiacus] and tree shrews [Tupaia belangeri]),

291 are capable of minimal viral replication while others (big brown bats [Eptesicus fuscus]) do not 292 appear to become infected at all, which suggests that while the virus may have originated in bats, 293 they are unlikely to serve as reservoir hosts $(30,31,32)$. The confounding clinical response to 
for reservoir maintenance difficult. Despite best attempts to predict host susceptibility based on receptor similarity or other modeling approaches, experimental infections remain the gold standard for evaluating the susceptibility of an animal to infection and following the course of disease.

Our results demonstrate that several common peridomestic wildlife species, including deer mice, bushy-tailed woodrats, and striped skunks are susceptible to SARS-CoV-2 infection and can shed infectious virus. Importantly, our work and the work of others indicate that so far, the majority of exposed wildlife species develop mild to no clinical disease and either fail to shed virus at all or shed low levels for short durations. Perhaps equally important is that these experimental infections suggest that we can rule out several common rodents, select wild lagomorphs and raccoons as potential SARS-CoV-2 reservoirs. There are, however, limitations to these experimental models, namely that the animals in our studies were directly exposed to high doses (e.g., $5 \log _{10} \mathrm{pfu}$ ) of virus, which is unlikely to be representative of an exposure in nature. Additionally, experimental infections using low numbers of apparently healthy, immunocompetent animals do not generate sufficient data to fully characterize the risk posed to animals of varying ages and health status. However, the results of this work and the work of others, combined with the dramatic response to infection seen in certain species such as mink, indicate that the possibility exists of SARS-CoV-2 infecting wildlife, establishing a transmission cycle, and becoming endemic in non-human species. In particular, the relatively high titers observed in select woodrat tissues (e.g., $5.2 \log _{10} \mathrm{pfu} / \mathrm{gram}$ of lung) suggests that a predator-prey transmission scenario among this rodent species and various small wild and domestic carnivore species is plausible. The major outcomes of such an event include direct threat to the health of wildlife and establishment of a reservoir host, which could complicate control measures of this 
virus in human populations. Experimental studies to identify and characterize species' response to SARS-CoV-2 infection help scientists classify those species that are at highest risk and allow for the implementation of prevention measures. For example, both deer mice and bushy-tailed

321 woodrats are commonly found in barns and sheds in very close proximity to humans, so when

322 cleaning out sheds or attempting to rodent-proof barns, people should consider wearing

323 appropriate personal protective equipment, both to prevent exposure to the pathogens rodents

324 carry as well as to prevent exposing wildlife to SARS-CoV-2. Likewise, humans with COVID19

325 who also own cats and dogs should practice extra precaution with their pets, including

326 minimizing the pet's exposure to wildlife. Notably, a photo-monitoring study provided evidence

327 that striped skunks can commonly use the same urban cover types (e.g., outbuildings and decks)

328 as domestic cats (33). Intentionally available pet food and spilled bird feed, which were two of

329 the attractants evaluated, produced instances where skunks and domestic cats were documented

330 to be on study sites simultaneously or nearly simultaneously, which could facilitate interspecies transmission of SARS-CoV-2.

Wildlife and SARS-CoV-2 are intricately involved, from the initial spillover event to potential reverse zoonosis, and we will undoubtedly continue to discover more susceptible species as the search for zoonotic reservoirs continues. COVID19 is just the latest in a series of examples of how the human-wildlife interface continues to drive the emergence of infectious disease. The use of experimental research, surveillance, and modeling as tools for predicting

337 outbreaks and epidemics will hopefully provide the knowledge base and resources necessary to prevent future pandemics. 


\section{Acknowledgments}

Department of Agriculture, Animal and Plant Health Inspection Service. A. Walker and L.

344 Guilbert were supported by the USDA Animal Health and Disease Veterinary Summer Scholar's

345 Program.

346

347

348

349

350

351

352

353

354

355

356

357

358

359

360

361

This work was funded by internal funding from Colorado State University and the U.S.

\section{Disclaimers}

None

\section{Author Bio}

Angela Bosco-Lauth is an Assistant Professor in the Department of Biomedical Sciences at Colorado State University. Here research interests include pathogenesis, transmission and ecology of infectious disease.

\section{References}

1. Zhou P, Yang X-L, Wang X-G, Hu B, Zhang L, Zhang W, et al. A pneumonia outbreak associated with a new coronavirus of probable bat origin. Nature. 2020 Mar $1 ; 579(7798): 270-3$.

2. Root JJ, Bosco-Lauth AM, Bielefeldt-Ohmann H, Bowen RA. Experimental infection of peridomestic mammals with emergent H7N9 (A/Anhui/1/2013) influenza A virus:

Implications for biosecurity and wet markets. Virology. 2016 Jan;487:242-8.

3. Shriner SA, VanDalen KK, Mooers NL, Ellis JW, Sullivan HJ, Root JJ, et al. LowPathogenic Avian Influenza Viruses in Wild House Mice. Davis T, editor. PLoS ONE. 2012 Jun 15;7(6):e39206. 
4. Romero Tejeda A, Aiello R, Salomoni A, et al. Susceptibility to and transmission of H5N1 and H7N1 highly pathogenic avian influenza viruses in bank voles (Myodes glareolus). Vet Res 46, 51 (2015).

5. Martínez-Hernández F, Isaak-Delgado AB, Alfonso-Toledo JA, Muñoz-García CI, Villalobos G, Aréchiga-Ceballos N, et al. Assessing the SARS-CoV-2 threat to wildlife: Potential risk to a broad range of mammals. Perspectives in Ecology and Conservation. 2020 Oct;18(4):223-34.

6. Luan J, Lu Y, Jin X, Zhang L. Spike protein recognition of mammalian ACE2 predicts the host range and an optimized ACE2 for SARS-CoV-2 infection. Biochemical and Biophysical Research Communications. 2020 May;526(1):165-9. range of SARS-CoV-2 predicted by comparative and structural analysis of ACE2 in vertebrates. Proc Natl Acad Sci USA. 2020 Sep 8;117(36):22311-22. Honing RW, et al. SARS-CoV-2 infection in farmed minks, the Netherlands, April and

9. DeLiberto T and Shriner S. Coronavirus disease 2019 update (536): Animal, USA (Utah), wild mink, first case. ProMED-mail, International Society for Infectious Diseases. Posted December 13, 2020. E, et al. Clinical and Pathological Findings in SARS-CoV-2 Disease Outbreaks in Farmed Mink ( Neovison vison ). Vet Pathol. 2020 Sep;57(5):653-7. 

coronavirus-2 natural animal reservoirs and experimental models: systematic review. Rev Med Virol [Internet]. 2020 Nov 18 [cited 2021 Jan 18]; Available from: https://onlinelibrary.wiley.com/doi/10.1002/rmv.2196 transmission of SARS-CoV-2 in golden hamsters. Nature. 2020 Jul;583(7818):834-838. Roborovski dwarf hamster is a highly susceptible model for a rapid and fatal course of SARS-CoV-2 infection. Cell Rep. 2020 Dec 8;33(10):108488. infection, neuropathogenesis and transmission among deer mice: Implications for reverse zoonosis to New World rodents [Internet]. Microbiology; 2020 Aug [cited 2020 Dec 7]. Available from: http://biorxiv.org/lookup/doi/10.1101/2020.08.07.241810 American deer mice are susceptible to SARS-CoV-2 [Internet]. Microbiology; 2020 Jul [cited 2020 Dec 14]. Available from: http://biorxiv.org/lookup/doi/10.1101/2020.07.25.221291 10(1): $1-7$. 
transmission, and response to reexposure in cats. Proc Natl Acad Sci USA. 2020 Oct 20;117(42):26382-8.

18. Shi J, Wen Z, Zhong G, Yang H, Wang C, Huang B, et al. Susceptibility of ferrets, cats, dogs, and other domesticated animals to SARS-coronavirus 2. Science. 2020 May 29;368(6494):1016-20.

19. Gross J, Elvinger F, Hungerford LL, Gehrt SD. Raccoon use of the urban matrix in the Baltimore Metropolitan Area, Maryland. Urban Ecosyst. 2012 Sep;15(3):667-82. Population in a Suburban Residential Community. Journal of Mammalogy. 1977 Nov 29;58(4):623-36. America: A conceptual model for perpetuation of the pathogen. Science of The Total Environment. 2020 Sep;733:139358. 2019 novel coronavirus (2019-nCoV) by real-time RT-PCR. Eurosurveillance [Internet]. 2020 Jan 23 [cited 2020 Nov 28];25(3). CoV-2 infection and the potential for reinfection. Journal of General Virology. 2020 Aug 1;101(8):791-7.

24. Patterson EI, Elia G, Grassi A, Giordano A, Desario C, Medardo M, et al. Evidence of exposure to SARS-CoV-2 in cats and dogs from households in Italy. Nat Commun. 2020 Dec;11(1):6231. 
25. de Morais HA, dos Santos AP, do Nascimento NC, Kmetiuk LB, Barbosa DS, Brandão PE, et al. Natural Infection by SARS-CoV-2 in Companion Animals: A Review of Case Reports and Current Evidence of Their Role in the Epidemiology of COVID-19. Front Vet Sci. 2020 Oct 27;7:591216.

26. Hammer AS, Quaade ML, Rasmussen TB, Fonager J, Rasmussen M, Mundbjerg K, et al. SARS-CoV-2 Transmission between Mink ( Neovison vison ) and Humans, Denmark. Emerg Infect Dis [Internet]. 2021 Feb [cited 2020 Dec 7];27(2).

27. Kim Y-I, Kim S-G, Kim S-M, Kim E-H, Park S-J, Yu K-M, et al. Infection and Rapid Transmission of SARS-CoV-2 in Ferrets. Cell Host \& Microbe. 2020 May;27(5):704709.e2.

28. Freuling CM, Breithaupt A, Müller T, Sehl J, Balkema-Buschmann A, Rissmann M, et al. Susceptibility of Raccoon Dogs for Experimental SARS-CoV-2 Infection. Emerg Infect Dis. 2020 Dec;26(12):2982-5.

29. Cohen J. From mice to monkeys, animals studied for coronavirus answers. Science. 2020 Apr 17;368(6488):221-2.

30. Hall JS, Knowles S, Nashold SW, Ip HS, Leon AE, Rocke T, et al. Experimental challenge of a North American bat species, big brown bat (Eptesicus fuscus ), with SARS-CoV-2. Transboundary and Emerging Diseases. 2020 Dec 9;tbed.13949.

31. Schlottau K, Rissmann M, Graaf A, Schön J, Sehl J, Wylezich C, et al. SARS-CoV-2 in fruit bats, ferrets, pigs, and chickens: an experimental transmission study. The Lancet Microbe. 2020 Sep;1(5):e218-25.

32. Zhao Y, Wang J, Kuang D, Xu J, Yang M, Ma C, et al. Susceptibility of tree shrew to SARS-CoV-2 infection. Sci Rep. 2020 Dec;10(1):16007. 
33. Weissinger MD, Theimer TC, Bergman DL, Deliberto TJ. Nightly and seasonal

Address for correspondence: Angela Bosco-Lauth, Department of Biomedical Sciences,

457 Colorado State University, 1683 Campus Delivery, Fort Collins, CO 80523. email:

$458 \quad$ angela.bosco-lauth@,colostate.edu 